\title{
Antibacterial Activity of Black Tea Extract against S. mutans, S. aureus, L. acidophilus, Klebsiella and E. coli
}

\author{
Pinky Goswami ${ }^{1}$, Chandana Kalita², Atool Chandra Bhuyan³ \\ ${ }^{1}$ Department of Dentistry, JMCH, Jorhat, Assam, India. ${ }^{2}$ Department of Conservative Dentistry, RDC, Guwahati, Assam, \\ India. ${ }^{3}$ Department of Conservative Dentistry, RDC, Guwahati, Assam, India.
}

\section{ABSTRACT}

\section{BACKGROUND}

Drinking tea has antioxidant, anti-inflammatory and anti-carcinogenic properties. In addition, tea is also considered beneficial for cardiovascular health and oral health. Health benefits of green tea are attributed to its polyphenol content. Catechins are the major polyphenols in green tea and in black tea the catechins are oxidized to theaflavins. Polyphenols present in tea have exhibited antimicrobial effects against a wide range of pathogenic bacteria. Studies have shown that green tea catechins are bioavailable in both plasma and urine. The aim of the present study is to evaluate the antibacterial activity of black tea extract against standard strains of $S$. mutans, $S$. aureus, L. acidophilus, Klebsiella and E. coli.

\section{METHODS}

Black tea extract was prepared by boiling black tea leaves in distilled water. It was then filtered, and the filtrate was treated with chloroform and ethyl acetate. The ethyl acetate was evaporated in a rotary evaporator and a brown coloured residue was obtained. Its antibacterial activity was studied against standard strains of five common bacteria i.e.; S. mutans, L. acidophilus, S. aureus, Klebsiella spp, and E. coli and the MIC of the black tea extract was determined using serial dilution method.

\section{RESULTS}

Black tea extract showed sufficient antibacterial activity against the tested bacteria. The MIC of black tea extract was lowest against Staphylococcus aureus.

\section{CONCLUSIONS}

Tea extracts have significant antimicrobial activity at varying concentrations against different bacterial pathogens.

\section{KEY WORDS}

Black Tea Extract, Antibacterial Activity, Catechins, Theaflavins
Corresponding Author:

Dr. Pinky Goswami,

Assistant Professor

Department of Dentistry,

JMCH, Jorhat, Assam, India.

E-mail: pinkyjmcjorhat@gmail.com

DOI: $10.14260 /$ jemds $/ 2020 / 5$

Financial or Other Competing Interests: None.

How to Cite This Article:

Goswami P, Kalita C, Bhuyan AC. Antibacterial activity of black tea extract against S. mutans, S. aureus, L. acidophilus, klebsiella and E. coli. J. Evolution Med. Dent. Sci. 2020;9(01):18-22, DOI: 10.14260/jemds/2020/5

Submission 09-11-2019,

Peer Review 21-12-2019,

Acceptance 27-12-2019,

Published 06-01-2020.

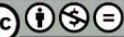




\section{BACKGROUND}

Tea is obtained from the plant Camellia sinensis which belongs to theaceae family. There are two major types of tea plants; China variety (Camellia sinensis sinensis) and Assam variety (Camellia sinensis assamica). A third Cambodian variety is also recognized but it is not cultivated. There are four major types of tea, namely black tea, green tea, oolong tea and white tea.

The manufacturing process of tea involves three basic steps called withering, fixing and rolling. The process of withering reduces the moisture content of the tea leaves and it allows the flavour compounds to develop. Fixing refers to the process by which enzymatic browning of the wilted leaves is controlled through the application of heat. During the manufacture of green tea, the fermentation of tea leaves is prevented by applying heat. With no fermentation, green tea leaves retain their green colour and almost all of their original polyphenol content. White tea is minimally processed and is obtained from the unopened buds or from the first shoot of the plant. Oolong tea is semi-fermented while black tea is fully fermented (1).The different processes of manufacturing give the various teas their characteristic colours and flavours.

Green tea contains a type of colourless molecule called catechins. The catechins belong to a group of chemicals called polyphenols. The polyphenols are of two types: primary polyphenols and secondary polyphenols. Primary polyphenols are classified as flavan-3-ols and hydrolysable tannins. (2) Catechins are flavan-3-ols. Secondary polyphenols are formed by oxidation of catechins during fermentation of tea leaves. Secondary polyphenols include theaflavins, theaflagillins and theasinensis.(2) There are four major catechins in green tea: epicatechin (EC), epigallocatechin (EGC), epicatechin gallate (ECG) and epigallocatechin gallate(3) EGCG is the most abundant catechin and comprises of approximately $59 \%$ of the total catechins. EGC, ECG and EC are present in $19 \%, 13.6 \%$ and $6.4 \%$ respectively. ${ }^{(3)}$ The major theaflavins of black tea are theaflavin, theaflavin 3gallate, theaflavin 3'-gallate, and theaflavin 3,3'-gallate.(4) Theaflavins are orange-red compounds responsible for the astringent taste and coppery colour of black tea.(5)

Mc Naught,(6) a major in the British Army Medical Corps first demonstrated the anti-microbial activity of tea. He showed that black tea infusion killed Salmonella typhi and Brucella melitensis. He suggested that the troops should carry tea in their bottles to prevent the outbreak of infections caused by these organisms. However proper scientific investigation of the antimicrobial activity of tea began only in the late 1980's and studies have verified that tea can kill and inhibit a wide range of pathogenic bacteria.

Tea is a rich source of polyphenols and studies have shown that these polyphenols possess antioxidant, antiviral and anti-inflammatory activities. Among all tea polyphenols, epigallocatechin gallate (EGCG) has been found to be responsible for much of the health-promoting ability of green tea. ${ }^{(7)}$

In recent years, theaflavins of black tea have also attracted considerable interest, as they are shown to have various physiological actions, including antioxidant,(8) anticancer,(9) anti-atherosclerotic,(10) anti-inflammatory,(11) antiviral,(12) and anti-periodontitis(13) effects, as well as the prevention of osteoporosis(14). Furthermore, these compounds have been shown to have human health benefits including glucose-lowering(15) and anti-obesity actions,(16) as a prevention of lifestyle-related diseases. In addition, theaflavin and thearubigin has also shown to possess antibacterial properties both in-vivo and in-vitro.(17)

The present study was carried out to study the antibacterial activity of black tea extract against five common bacteria. The standard strains of Streptococcus mutans, Staphylococcus aureus, Lactobacillus acidophilus, Klebsiella pneumoniae \& E. coli were taken for the proposed study.

\section{METHODS}

Standard strains of the following types of bacteria were usedStreptococcus mutans (MTCC 497), Lactobacillus acidophilus (ATCC 43121) Staphylococcus aureus (ATCC 25923), Klebsiella pneumoniae (ATCC 700603) \& E. coli (ATCC 25922)

\section{Culture Media}

MSF Agar Medium, Tomato Juice Agar Medium, Nutrient Broth, Agar Plate.

\section{Method of Preparing Black Tea Extract}

$12 \mathrm{gm}$ of black tea was boiled in $90 \mathrm{ml}$ of distilled water for ten minutes and then filtered with Whatman No.1 filter paper. The filtrate was subsequently treated with chloroform and ethyl acetate. The ethyl acetate was evaporated in a rotary evaporator and a dark brown residue was obtained. The residue was dissolved in $3 \mathrm{ml}$ of normal saline.(18)

\section{Culturing of Freeze-Dried $S$. mutans}

The freeze-dried S. mutans (MTCC 497) obtained from IMTECH (Institute of Microbial Technology, Chandigarh) was first transferred to brain heart infusion (BHI) broth and incubated for 24 hours to make the $S$. mutans viable.(19) For isolation of Lactobacillus tomato juice agar medium was used. Further subculture was done in peptone water enriched with serum. For isolation of Staphylococcus, Klebsiella and E. coli nutrient broth was used as a medium. Antibacterial activity against the mentioned bacteria was performed by using agar well diffusion method.(20)

After observation of antibacterial activity, the MIC was determined using broth dilution method.(21) The serial dilution was used as a two-fold dilution for proper demarcation of the end point. Two-fold dilution of the tea extract was made starting from 1:20 up to 1:2560.

\section{RESULTS}

1. The minimum inhibitory concentration of black tea against $S$. mutans was found to be $3.68 \mathrm{mg} / \mathrm{ml}$.

2. The minimum inhibitory concentration of black tea against Lactobacillus was found to be $14.72 \mathrm{mg} / \mathrm{ml}$.

3. The minimum inhibitory concentration of black tea against Staphylococcus was found to be $1.42 \mathrm{mg} / \mathrm{ml}$.

4. The minimum inhibitory concentration of black tea against $E$. coli was found to be $3.68 \mathrm{mg} / \mathrm{ml}$. 
5. The minimum inhibitory concentration of black tea against Klebsiella was found to be $14.72 \mathrm{mg} / \mathrm{ml}$.

\begin{tabular}{|c|c|c|c|c|c|c|c|c|c|}
\hline & $1: 10$ & $1: 20$ & $1: 40$ & $1: 80$ & $1: 160$ & $1: 320$ & $1: 640$ & $1: 1280$ & $1: 2560$ \\
\hline S. mutans (one) & - & - & - & - & + & + & + & + & + \\
\hline S. mutans (two) & - & - & - & + & + & + & + & + & + \\
\hline S. mutans (three & - & - & - & + & + & + & + & + & + \\
\hline S. mutans (four) & - & - & - & + & + & + & + & + & + \\
\hline S. mutans (five) & - & - & - & + & + & + & + & + & + \\
\hline S. mutans (six) & - & - & + & + & + & + & + & + & + \\
\hline Table 1. Se & rial Di & ilution & of $T e$ & a Extr & $\begin{array}{l}\text { ract ag } \\
\text { iple Dil }\end{array}$ & $\begin{array}{l}\text { gainst S } \\
\text { lution }\end{array}$ & S. muta & ans Sam & ples. \\
\hline
\end{tabular}

\begin{tabular}{|c|c|c|c|c|c|c|c|c|c|}
\hline & $1: 10$ & $1: 20$ & $1: 40$ & $1: 80$ & $1: 160$ & $1: 320$ & $1: 640$ & 01:128 & \begin{tabular}{l|l|}
$01: 2560$ \\
\end{tabular} \\
\hline Lactobacillus (one) & - & - & - & - & + & + & + & + & + \\
\hline Lactobacillus (two) & - & - & - & + & + & + & + & + & + \\
\hline Lactobacillus (three) & - & - & - & + & + & + & + & + & + \\
\hline Lactobacillus (four) & - & - & - & + & + & + & + & + & + \\
\hline Lactobacillus (five) & - & - & - & + & + & + & + & + & + \\
\hline Lactobacillus (six) & - & - & + & + & + & + & + & + & + \\
\hline Table $2 . S$ & $\begin{array}{r}\text { rial } \\
\text { Sa }\end{array}$ & uti & of & $a E x$ & $\begin{array}{l}\text { xtract } \\
\text { ample }\end{array}$ & agains & $\begin{array}{l}\text { st Lact } \\
\text { ion }\end{array}$ & tobacillt & \\
\hline
\end{tabular}

\begin{tabular}{|l|c|c|c|c|c|c|c|c|c|}
\hline & $1: 10$ & $1: 20$ & $1: 40$ & $1: 80$ & $1: 160$ & $1: 320$ & $1: 640$ & $1: 1280$ & $1: 2560$ \\
\hline Staphylococcus(one) & - & - & - & - & + & + & + & + & + \\
\hline Staphylococcus (two) & - & - & - & - & + & + & + & + & + \\
\hline Staphylococcus(three) & - & - & - & - & + & + & + & + & + \\
\hline Staphylococcus (four) & - & - & - & - & + & + & + & + & + \\
\hline Staphylococcus (five) & - & - & - & - & + & + & + & + & + \\
\hline Staphylococcus (six) & - & - & - & - & + & + & + & + & + \\
\hline
\end{tabular}

\begin{tabular}{|c|c|c|c|c|c|c|c|c|c|}
\hline & $1: 10$ & $1: 20$ & $1: 40$ & $1: 80$ & $1: 160$ & $1: 320$ & $1: 640$ & $1: 1280$ & $1: 2560$ \\
\hline E. coli (one) & - & - & - & + & + & + & + & + & + \\
\hline E. coli (two) & - & - & - & + & + & + & + & + & + \\
\hline E. coli (three) & - & - & - & + & + & + & + & + & + \\
\hline E. coli (four) & - & - & - & + & + & + & + & + & + \\
\hline E. coli (five) & - & - & - & + & + & + & + & + & + \\
\hline E. coli (six) & - & - & - & + & + & + & + & + & + \\
\hline
\end{tabular}

\begin{tabular}{|c|c|c|c|c|c|c|c|c|c|}
\hline & $1: 10$ & $1: 20$ & $1: 40$ & $1: 80$ & $1: 160$ & $1: 320$ & $1: 640$ & $1: 1280$ & $1: 2560$ \\
\hline Klebsiella (one) & - & - & + & + & + & + & + & + & + \\
\hline Klebsiella (two) & - & - & + & + & + & + & + & + & + \\
\hline Klebsiella (three) & - & - & + & + & + & + & + & + & + \\
\hline Klebsiella (four) & - & - & + & + & + & + & + & + & + \\
\hline Klebsiella (five) & - & + & + & + & + & + & + & + & + \\
\hline Klebsiella (six) & - & + & + & + & + & + & + & + & + \\
\hline
\end{tabular}

Table 5. Serial Dilution of Tea Extract against Klebsiella Samples. Bacterial Sample Dilution

\section{DISCUSSION}

Chemical composition analysis has increased dramatically in the last decade due to an abundance of scientific data regarding the positive effect of tea on human health. Epidemiological and animal studies suggest that tea is protective against certain cancers, cardiovascular diseases, and neurodegenerative diseases (Yang \& Koo, 2000; Mandel \& Youdim, 2004; Butt et al., 2015)

The majority $(75 \%)$ of the tea consumed all over the world is black tea. Both black tea and green tea are reportedly good for human health. Theaflavins are a group of polyphenols unique to black tea. They are formed during the fermentation process and comprise $3-6 \%$ of all polyphenols in black tea. Theaflavins possess many health benefits. In fact some studies have shown that theaflavin 3, 3' - gallate have higher antioxidant activity than EGCG which is the strongest anti-oxidant among the green tea catechins.(22) The anti- oxidant properties of theaflavins have been attributed to their gallic acid moiety.(23) Theaflavins have more $\mathrm{OH}$ groups than catechins since theaflavins are dimmers of catechins.(24) Tea polyphenols are known for their antibacterial activity.

In the present study the antibacterial activity of black tea extract was studied against five common bacteria. Out of these, Streptococcus mutans and Lactobacillus are involved in the aetiology of dental caries. S aureus causes skin infections, pneumonia, heart valve infections and bone infections. Klebsiella causes pneumonia, UTI and meningitis and E. coli is a common uropathogen. It was observed from the present study that the black tea extract showed sufficient antibacterial activity against $S$. mutans, $S$. aureus, $L$. acidophilus, Klebsiella spp. and E. coli. The MIC of Black tea extract against $S$. mutans was $3.68 \mathrm{mg} / \mathrm{ml}$, the MIC against $S$. aureus was $1.42 \mathrm{mg} / \mathrm{ml}$, and the MIC against Lactobacillus was $14.72 \mathrm{mg} / \mathrm{ml}$.

Sakanaka et al.(25) studied the effects of Japanese green tea extract on Streptococcus mutans. They found that green tea extract inhibited the growth of $S$. mutans. The analysis of the extract revealed that the main antibacterial components were several polyphenolic compounds and, epigallocatechin gallate, was the most active component and its minimum inhibitory concentration against the bacterium was found to be $250 \mathrm{mg} / \mathrm{ml}$.

Yam and Shah (26) studied the microbiological activity of crude and fractionated crude extracts of tea (Camellia sinensis). They found that aqueous extracts of different types of tea inhibited a wide range of pathogenic bacteria, including methicillin-resistant Staphylococcus aureus. Tea extracts were found to be bactericidal against Staphylococci and Yersinia enterolytica at well below cup of tea concentration. They confirmed that the antibacterial activity of green tea can be explained by its content of epicatechin, epigallocatechin gallate and epicatechin gallate. In black tea extracts, theaflavin and its gallates are the active antibacterial compounds.

In a study carried out by $\mathrm{Xin} \mathrm{Xu}$, et al(27) it was observed that green tea catechin epigallocatechin gallate inhibited the growth of $S$. mutans. The MIC was $31.25 \mu \mathrm{g} / \mathrm{mL}$ and the minimum bactericidal concentration was $62.5 \mu \mathrm{g} / \mathrm{mL}$. Wanda Reygaert et al(28) studied the effect of green tea extract on uropathogenic $E$. coli and found that all of the strains tested (except one) showed an MIC of $\leq 4.0 \mathrm{mg} / \mathrm{ml}$. They concluded that among the green tea catechins, EGC was excreted in urine in high enough concentration to be effective as an antimicrobial against infection caused by E. coli.

Abhishek Mehta, et al(29) carried out a comparative evaluation of antibacterial activity of Aqueous, Ethanolic, Methanolic and Acetone extracts of Commercial Green Tea and Black Dust Tea against three Standard (ATCC) bacterial strains: 1)Staphylococcus aureus ATCC 25923 2)Escherichia coli ATCC 25922 3)Pseudomonas aeruginosa ATCC 27853 by Agar well Diffusion method. S. aureus was found to be the most susceptible to tea extracts followed by $E$. coli and $P$. aeruginosa. Amongst all tea extracts tested the aqueous extract of Green tea has shown the highest antimicrobial activity against $S$. aureus ATCC 25922 . Similar results were obtained in the present study and the black tea extract was found to possess the lowest MIC against $S$. aureus (ATCC 25922). Black tea extract was tested against five different types of bacteria and out of these, S. aureus was most 
susceptible followed by S. mutans and E. coli. Klebsiella and $L$. acidophilus were least susceptible.

Some authors have postulated that tea catechins exert their anti-bacterial activity by different mechanisms like changes in cell permeability, by modification of intercellular functions or by modification in cell wall rigidity. $(30,31)$ Gramza and Korczak ${ }^{(32)}$ hypothesized that antimicrobial activity of tea extracts could be due to the fact that the negatively charged EGCG binds strongly to the positively charged lipid bilayer of Gram- positive bacteria. Catechins partitioning in the lipid bilayer membrane result in loss of cell structure and function and finally result in cell death. Bacterial cell membrane damage inhibits the ability of the bacteria to bind to host cells and to each other to form biofilms. Bacterial cell membrane damage also results in an inability of the bacteria to secrete toxins. Other researchers have also pointed out that the antibacterial effect of catechins could be due to their inhibitory effect on certain proteins (33) or it may also be due to iron deprivation or hydrogen binding with vital proteins such as microbial enzymes.(34) These supports the fact that polyphenols are responsible for the antimicrobial activities of the tea extracts.

Although numerous studies have been conducted with green tea catechins, there are very few studies on theaflavins because they are present in extremely low concentration in black tea and their extraction is difficult.(35) Research is being carried out to develop biosynthetic methods for the mass production of theaflavins.

\section{CONCLUSIONS}

Tea extracts have significant antimicrobial activity at varying concentrations against different bacterial pathogens.

\section{REFERENCES}

[1] Jigisha A, Nishant R, Navin K, et al. Green tea: a magical herb with miraculous outcomes. Int Res J Pharm 2012;3(5):139-48.

[2] Sang S, Yang CS, Ho CT. Peroxidase mediated oxidation of catechins. Phytochem Rev 2004;3(1-2):229-41.

[3] Cabrera C, Artacho R, Gimenez R. Beneficial effects of green tea- a review. J Am Coll Nutr 2006;25(2):79-99.

[4] Collier PD, Bryce T, Mallows R, et al. The theaflavins of black tea. Tetrahedron 1973;29:125-42.

[5] Łuczaj W, Skrzydlewska E. Antioxidative properties of black tea. Prev Med 2005;40(6):910-18.

[6] McNaught JG. On the action of cold or lukewarm tea on Bacillus typhosus. Journal of the Royal Army Medical Corps 1906;7:372-3.

[7] Gupta DA, Bhaskar DJ, Gupta RK, et al. Green tea: a review of its natural anti-oxidant therapy and cariostatic benefits. Biol Sci Pharm Res 2014;2:8-12.

[8] Peluso I, Serafini M. Antioxidants from black and green tea: from dietary modulation of oxidative stress to pharmacological mechanisms. $\mathrm{Br} \mathrm{J}$ Pharmacol 2017;174(11):1195-1208.
[9] Sur S, Panda CK. Molecular aspects of cancer chemopreventive and therapeutic efficacies of tea and tea polyphenols. Nutrition 2017;43-44:8-15.

[10] Loke WM, Proudfoot JM, Hodgson JM, et al. Specific dietary polyphenols attenuate atherosclerosis in apolipoprotein E-knockout mice by alleviating inflammation and endothelial dysfunction. Arterioscler Thromb Vasc Biol 2010;30(4):749-57.

[11] Wu Y, Jin F, Wang $Y$, et al. In-vitro and in-vivo antiinflammatory effects of theaflavin -3,3'-digallate on lipopolysaccharide-induced inflammation. Eur J Pharmacol 2017;794:54-60.

[12] Ohba M, Oka T, Ando T, et al. Antiviral effects of theaflavins against calciviruses. J Antibiot (Tokyo) 2017;70(4):443-7.

[13] Kong L, Qi X, Huang S, et al. Theaflavins inhibit pathogenic properties of $\mathrm{P}$. gingivalis and MMPs production in in P. gingivalis stimulated human gingival fibroblasts. Arch Oral Biol 2015;60(1):12-22.

[14] Oka Y, Iwai S, Amano H, et al. Tea polyphenols inhibit rat osteoclast formation and differentiation. J Pharmacol Sci 2012;118(1):55-64.

[15] Miyata Y, Tamaru S, Tanaka T, et al. Theaflavins and theasinensin A derived from fermented tea have antihyperglycemic and hypotriacylglycerolemic effects in KK-A mice and Sprague-Dawley rats. J Agric Food Chem 2013;61(39):9366-72.

[16] Takemoto M, Takemoto H, Saijo R. Theaflavin synthesized in a selective, domino type, one- pot enzymatic biotransformation method with Camellia sinensis cell culture inhibits weight gain and fat accumulation to high- fat diet-induced obese mice. Biol Pharm Bull 2016;39(8):1347-52.

[17] Jin Y, Jin CH, Row KH. Separation of catechin compounds from different teas. Biotechnol J 2006;1(2):209-13.

[18] Suramaniam P, Eswara U, Reddy MKR. Effect of different types of tea on Streptococcus mutans: an in vitro study. Ind J Dent Res 2012;23(1):43-8.

[19] Bancirova M. Comparison of the antioxidant capacity and the anti-microbial activity of black and green tea. Food Res Int 2010;43(5):1379-82.

[20] Bauer AW, Kirby WM, Sheris JC, et al. Antibiotic susceptibility testing by a standardized single disk method. Am J Clin Pathol 1966;45(4):493-6.

[21] Fani FM, Kahanteb J. Inhibitory activity of Aloe vera gel on some clinically isolated cariogenic and periodontopathic bacteria. J Oral Sci 2012;54(1):15-21.

[22] Leung LK, Su Y, Chen R, et al. Theaflavins in green tea and catechins in green tea are equally effective antioxidants. J Nutr 2001;131(9):2248-51.

[23] Shiraki M, Hara Y, Osawa T, et al. Antioxidative and antimutagenic effects of theaflavins from black tea. Mutat Res 1994;323(1-2):29-34.

[24] Miller NJ, Casteluccio C, Tijburg L, et al. The anti-oxidant properties of theaflavins and their gallate esters-radical scavengers or metal chelators? FEBS Lett 1996;392(1):40-4. 
[25] Sakanaka S, Kim M, Taniguchi M. Antibacterial substances in Japanese green tea extract against Streptococcus mutans, a cariogenic bacterium. Agr Biol Chem 1989;53(9):2307-11.

[26] Yam TS, Shah S, Hamilton-Miller JM. Microbiological activity of whole and fractionated crude extracts of tea (Camellia sinensis), and of tea components. FEMS Micbiol Lett 1997;152(1):169-74.

[27] Xu X, Zhou XD, Wu CD. The tea catechin Epigallocatechin gallate suppresses cariogenic virulence factors of Streptococcus mutans. Antimicrob Agents Chemother 2011;55(3):1229-36.

[28] Reygaert W, Jusufi I. Green tea as an effective antimicrobial for urinary tract infections caused by Escherichia coli. Front Microbial 2013;4:162.

[29] Mehta A, Saxena G, Mani A. Antimicrobial activity of ethanolic extract of commercial green tea against multi drug resistant bacterial strains. Int J Med Heal Res 2017;3(9):93-100.
[30] Ikigai H, Nakae T, Hara Y, et al. Bactericidal catechins damage the lipid bilayer. Biochim Biophys Acta 1993;1147(1):132-6.

[31] Taguri T, Tanaka T, Kouno I. Antibacterial spectrum of plant polyphenols and extracts depending upon hydroxyphenyl structure. Biol Pharm Bull 2006;29(11):2226-35.

[32] Gramza A, Korzak J. Tea constituents (Camellia sinesis) as antioxidants in lipid systems. Trends in Food Science and Technology 2005;16(8):351-8.

[33] Okamoto M, Leumg KP, Ansai T, et al. Inhibitory effects of green tea catechins on protein tyrosine phosphatase in Prevotella intermedia. Oral Microbiol Immunol 2003;18(3):192-5.

[34] Cushnie TP, Lamb AJ. Recent advances in understanding the antibacterial properties of flavonoids. Int J Antimicrob Agents 2011;38(2):99-107.

[35] Takemoto M, Takemoto H. Synthesis of theaflavins and their functions. Molecules 2018;23(4):918. 